\title{
Fair Resource Allocation in A Volatile Marketplace
}

\author{
MOHAMMAD HOSSEIN BATENI, Google Research \\ YIWEI CHEN, Singapore University of Technology and Design \\ DRAGOS FLORIN CIOCAN, INSEAD \\ VAHAB MIRROKNI, Google Research
}

We consider the setting where a seller must allocate a collection of goods to budgeted buyers, as exemplified by online advertising systems where platforms decide which impressions to serve to various advertisers. Such resource allocation problems are challenging for two reasons:

- The seller must strike a balance between optimizing her own revenues and guaranteeing fair allocations of goods to her buyers, incentivizing them to return to the platform in the future.

- In many settings, the supply of goods available to the seller evolves over time in unpredictable ways, making the allocation problem inherently dynamic.

We propose a stochastic approximation scheme akin to dynamic market equilibrium. Our scheme relies on frequent re-solves of an Eisenberg-Gale convex program, and does not require the seller to have any knowledge about how the goods arrival process evolves in the future. We show that our scheme satisfies the following properties:

- The algorithm clears buyer budgets even in the presence of uncertainty in the supply of goods, as long as the process governing this supply has continuous sample paths.

- Assuming that the supply process belongs to a natural family of stochastic processes that are weighted averages of Brownian motion shocks, we provide a 0.47 constant bound on the fairness loss our scheme incurs, relative to the optimal proportionally fair allocation that can be achieved with clairvoyant knowledge of the process' sample path. Our bound applies to processes (a) within a large parametric family and (b) of arbitrarily large volatility. Moreover, the family consists of non-stationary processes that are inherently difficult to learn: in fact, one can view our stochastic model as a middle ground between an iid/random permutation model and an adversarial one.

- In regimes when the volatility of the supply process goes to 0 , the gap becomes 0 ; in other words, our scheme is fairness optimal when the seller has reliable forecasts.

We then extend our results to a more general family of fairness metrics called $\alpha$-fairness. Finally, we deal with a multi-objective problem where the seller is concerned with both the proportional fairness and utilitarian efficiency of the allocation, and propose a hybrid algorithm which achieves a 0.27 bi-criteria guarantee against fairness and efficiency.

CCS Concepts: $\bullet$ Mathematics of computing $\rightarrow$ Stochastic processes; Stochastic control and optimization; $\bullet$ Theory of computation $\rightarrow$ Online algorithms; Market equilibria; $\bullet$ Applied computing $\rightarrow$ Economics;

Additional Key Words and Phrases: dynamic resource allocation; fairness; matching problems; network revenue management; online advertising

Link to complete paper - http://ssrn.com/abstract=2789380

Authors addresses: Mohammad Hossein Bateni, Google Research, email: bateni@google.com; Yiwei Chen, Singapore University of Technology and Design, email: yiwei_chen@sutd.edu.sg; Dragos Florin Ciocan, INSEAD, email: florin.ciocan@insead.edu; Vahab Mirrokni, Google Research, email: mirrokni@google.com.

Permission to make digital or hard copies of part or all of this work for personal or classroom use is granted without fee provided that copies are not made or distributed for profit or commercial advantage, and that copies bear this notice and the full citation on the first page. Copyrights for third-party components of this work must be honored. For all other uses, contact the owner/author(s). Copyright is held by the author/owner(s).

EC'16, July 24-28, 2016, Maastricht, The Netherlands.

ACM 978-1-4503-3936-0/16/07.

http://dx.doi.org/10.1145/2940716.2940763 